A CASE OF APPENDICITIS IN A CHILD: OPERATION ; RECOVERY.

BY ROYal WhitMan, M. D.,

Surgeon to the Orthopedic Department of the Boston Dispensary.

ON Saturday, July 16th, 1888, a boy of seven, having been in perfect health, on awakening after a restless night, complained of pain in the right side and of difficulty in moving the right leg. On Sunday he was worse; the pain more constant and severe, with much tenderness in the right flank. An enema was followed by two free movements of the bowels, he cried all night with pain, and on Monday I saw him. The patient appeared in fair condition, the abdomen not markedly swollen or tympanitic, the right inguinal region extremely sensitive to pressure, dull on percussion, and on palpation a round, hard tumor about the size of an orange was apparent; pulse 110, temperature $101^{\circ}$. The treatment was rest in bed, liquid diet, and sufficient morphine to control the pain, with a very small amount of which he was made comfortable for the two following days, with but little change in condition. On Thursday Dr. H. W. Cushing kindly saw him with me, and it was decided to wait another day for a change in the symptoms either for better or worse. On the following morning he was much worse, pulse 120 , temperature $102^{\circ}$, the abdomen swollen and tympanitic, the tumor somewhat larger, and better defined. Some delay was necessitated in procuring assistance, and on our arrival the mother informed us that in the interval the boy complained of such intense pain and his abdomen became so swollen that he could not breathe, so that in desperation she had given him a large enema, which had brought away foul-smelling shreds and white matter, which she had thrown away; a history of spontaneous opening of the abscess. An examination of the tumor showed no change in its outline; the temperature had gone up to $103.1^{\circ}$, and the pulse to 130 ; the patient was in a drowsy, stupid condition, from which he could be aroused only by the pain caused by examination. Some difficulty was experienced in deciding as to the proper treatment of the case. The probability of a spontaneous opening contraindicated an operation, and the surroundings were unfavorable to its after treatment. On the other hand, the patient was much worse, and it was decided that delay would be dangerous, especially on account of the time required to procure assistance in case the symptoms became more urgent. Accordingly, with the assistance of Drs. Cushing and Peckham the operation was performed, an incision about three inches in length being made over the outer third of Poupart's ligament. On dividing the abdominal muscles, the adherent wall of the abscess was opened, and about an ounce of foul pus evacuated. The cavity, which was about the size of a hen's egg, was thoroughly washed out with hot water, during which a seed, apparently of an orange, came away, two drainage-tubes .were inserted, and the remaining space packed with iodoform gauze. The after history of the case is uneventful: the temperature fell at once to normal, the packing and tubes were removed on the fourth day. During the absence of his mother, the child walked about the house on the fifth day; was dressed and on the street on the tenth day, when he was practically well, sixteen days after the first sign of the affection, though the wound did not completely close till a week later. He has since remained without a symptom to remind him of his illness. Several days after the operation the mother noticed a small amount of blood in the first dejection, making it probable that there had been an opening into the rectum which had immediately closed. The particular interest in this case is, I think, in the question as to whether or not, under the circumstances, an operation should have been performed. It might have been better to have made an examination by the rectum with the design of enlarging a possible opening. When, however, ether had been given, and the abdominal walls were relaxed, the tumor was found to fill the lower half of the right abdomen, so that the indications for an external opening seemed clear. In any event the result under a different treatment could hardly have proved more satisfactory than under that pursued.

\section{A CASE OF MACEWEN'S OPERATION}

(FOR THE RADICAL CURE OF INGUINAL HERNIA) IN A GIRL, RESULTING IN THE CURE OF HABITUAL INCONTINENCE OF URINE.

BY ROYAL WhITMAN, M.D.

THrs patient, a girl of eleven years of age, was brought to me last spring, more especially on account of an antero-posterior curvature of the spine. In addition she had a right inguinal hernia about the size of a small lemon, which had existed an indefinite time, and was the occasion of more or less discomfort; it was increasing in size, no attempt having been made to control it with a truss. There was also a history of habitual incontinence since infancy, making it impossible, as her mother expressed it, to keep her clothes in decent condition. As treatment by drugs had proved of little service, a radical cure of the hernia was advised, in the hope that the shock of the operation, with subsequent confinement to bed, might relieve this condition. On April 9th, 1888, Macewen's operation was performed, with the assistance of Dr. George Haven. The patient was about as usual on the eighteenth day without support, complete control of the urine having been established. Since then, her mother says, she has improved greatly both mentally and physically.

\section{Ifitportg of Sorietiex.}

\section{SURGICAL SECTION OF THE SUFFOLK} DISTRICT MEDICAL SOCIETY.

G. H. MONKS, M.D., SECRETARY.

MeEting, Wednesday evening, November 7th, at 8 o'clock. DR. J. C. WARREN presiding.

The Chairman having declined renomination, Dr. E. H. Bradford was, on the motion of Dr. M. H. Richardson, elected by acclamation Chairman of the Section for the ensuing year.

Dr. M. H. Richardson reported

TWO CASES OF LAPAROTOMY FOR EXTRA-UTERINE PREGNANCY. ${ }^{1}$

Dr. J. W. ELLIot said: I congratulate Dr. Richardson on his success. I think both cases are I Page 519 in Journal. 